\title{
Microinjection of the Monoclonal Anti-Tubulin Antibody YL1/2 Inhibits Cleavage of Sand Dollar Eggs
}

\author{
Mikako T. Oka ${ }^{1}$, Takao Arai ${ }^{2}$, and Yukihisa Hamaguchi ${ }^{+1}$ \\ ${ }^{1}$ Biological Laboratory, Faculty of Science, Tokyo Institute of Technology, Tokyo 152, and ${ }^{2}$ Department of \\ Applied Biological Science, Faculty of Science and Technology, Science University of Tokyo, Noda 278, Chiba, \\ Japan
}

Key words: cleavage/microinjection/mitotic apparatus/monoclonal antibody/sand dollar/tubulin

\begin{abstract}
Two monoclonal antibodies against $\alpha$-tubulin (YL1/2 and D2D6) were microinjected into the egg of the sand dollar Clypeaster japonicus, and their effects on cleavage of the egg were investigated. They had already been shown by immunobloting to react specifically with egg tubulin and by immunofluorescence to stain the mitotic apparatus [OKA et al., (1990). Cell Motil. Cytoskel. 16: 239-250]. Injection of YL1/2 prevented chromosome movement and cleavage, although the cleavage furrow developed in some cases. In all eggs injected at prometaphase, metaphase, or anaphase, the birefringence of the mitotic apparatus disappeared immediately after injection. Injection of D2D6 had no significant effect on mitosis or cleavage of whole eggs injected after nuclear disappearance, although it prevented the disappearance of the nuclear envelope in $54 \%$ of the eggs injected before the disappearance. FITC-conjugated D2D6 did not accumulate in the spindle when injected into the dividing sand dollar egg. These results indicate that YL1/2 disassembled microtubules, whereas D2D6 did not bind to microtubules in the living cell.
\end{abstract}

Microtubules play very important roles in mitotic mechanisms: Spindle and astral microtubules are involved in chromosome movement, spindle elongation, and the establishment of the cleavage plane. Recently we have revealed by immunofluorescence using monoclonal anti-tubulin antibodies that the tubulin isotypes of the spindle differ from those of the aster (13). Heterogeneity in spindle microtubules has been reported (5, $8,14,16,17,21)$. These findings substantiate the idea that functionally differentiated microtubules are composed of different tubulin isotypes $(1,2,4,7)$. Because monoclonal anti-tubulin antibodies are specific to tubulin isotypes, they are useful not only for studying microtubules in the fixed cell by immunofluorescence, but also for investigating microtubule function in the living cell. However, it is not clear whether they react with tubulin in the living cell: some monoclonal antitubulin antibodies were found to bind to microtubules when injected into living cells $(3,6,19,20)$, but others were not $(3,6)$.

In this study, two monoclonal antibodies, YL1/2 and D2D6, were injected into the sand dollar egg to examine the roles of microtubules in mitosis in vivo. That both reacted specifically with egg tubulin was determined by analyzing their reactivity by immunobloting, and that both stained the mitotic apparatus was demonstrated by

\footnotetext{
To whom correspondence should be addressed.
}

indirect immunofluorescence (13). We found that YL1/2 induced microtubule depolymerization and inhibited cell division, whereas D2D6 did not cause any significant effects.

\section{MATERIALS AND METHODS}

Materials. Eggs of the sand dollar Clypeaster japonicus were obtained by intracoelomic injection of $1 \mathrm{mM}$ acetylcholine in sea water, rinsed three times with artificial sea water (Jamarin U, Jamarin Laboratory, Osaka), and stored at $15^{\circ} \mathrm{C}$ until use. Sperm were collected as "dry", stored at $4^{\circ} \mathrm{C}$, and diluted in sea water just prior to use. Eggs were inseminated in a small volume of artificial sea water. After $1 \mathrm{~min}$, the egg suspension was pipetted gently after the addition of more than 10 times the volume of $1 \mathrm{M}$ urea solution to remove the fertilization envelope. After three rinses with Ca-free artificial sea water (Jamarin Laboratory, Osaka), the eggs were allowed to develop in Ca-free artificial sea water at $25^{\circ} \mathrm{C}$ and then removed into artificial sea water at injection.

YL1/2, a monoclonal antibody (ascites) against yeast $\alpha$ tubulin specific to tyrosinated $\alpha$-tubulin (11), and D2D6, a monoclonal antibody (ascites or culture supernatant) against squid nerve $\alpha$-tubulin (1), were used. Each antibody was precipitated with $50 \%$ saturated ammonium sulfate and dialyzed against a dilution solution $[10 \mathrm{mM}$ Tris and $140 \mathrm{mM} \mathrm{NaCl}$ ( $\mathrm{pH}$ 7.4)]. The protein concentrations of YL1/2, D2D6 ascites, and D2D6 culture supernatant were $39 \mathrm{mg} / \mathrm{ml}, 37$ 
$\mathrm{mg} / \mathrm{ml}$, and $13 \mathrm{mg} / \mathrm{ml}$, respectively. The protein concentrations were determined by Lowry's method (12). The dilution solution, $30 \mathrm{mg}$ rat $\mathrm{IgG} / \mathrm{ml}$, and $30 \mathrm{mg}$ mouse $\mathrm{IgG} / \mathrm{ml}$ were used as controls.

Methods. Injection was carried out with Narishige's micromanipulator of the oil pressure type (MO-102R) and its microinjector (IM-4A, Narishige Sci. Inst. Lab., Tokyo) as described by Hiramoto (10). The amount of injected antibody was $1-5 \%$ of the egg volume. The concentrations of YL1/2, D2D6 ascites, and D2D6 culture supernatant were calculated to be $2.6-12.9 \mu \mathrm{M}, 2.5-12.3 \mu \mathrm{M}$, and $0.9-4.3 \mu \mathrm{M}$, respectively, in the sand dollar egg, assuming that the total protein constituent of the injectate was antibody, whose molecular weight is 150,000 .

For conjugation with FITC, the antibody solution was mixed with $1 / 10$ th volume of a carbonate buffer $[0.15 \mathrm{M} \mathrm{NaCl}$ and $\left.0.5 \mathrm{M} \mathrm{NaCO}_{3}(\mathrm{pH} 9.5)\right]$ and $1 / 50$ th volume of $20 \mathrm{mg}$ FITC/ml DMSO, and incubated for $1 \mathrm{~h}$ at $4^{\circ} \mathrm{C}$ (18). Free FITC was removed by dialysis against the dilution solution.

Observation was with a Nikon OPTIPHOT equipped with differential interference (NT). In some cases, the same microscope equipped with epifluorescence or polarization optics was used. Photographs were taken on Panatomic-X or TMAX-100 and the former developed with Superprodol and the latter with T-MAX Developer.

\section{RESULTS}

Inhibition of cell division by microinjection of $Y L 1 / 2$. We microinjected the monoclonal antibody YL1/2 into $C$. japonicus eggs and investigated its effects on cell division. Cell division was inhibited in most eggs injected with YL1/2 as described below, whereas division occurred normally after injection of rat IgG solution or the dilution buffer. As shown in Figure 1, injecting $\mathrm{YL} 1 / 2$ into the egg before nuclear disappearance prevented that event in $94 \%$ of the injected eggs; this effect continued for at least 3 hours after injection. In some cases $(6 \%)$, the nuclear envelope disappeared after injection. However, the spindle did not form or the eggs did not cleave. Subsequently, one nucleus reappeared in these eggs.

Injecting YL1/2 at prometaphase inhibited chromosome movement and subsequently inhibited both nuclear and cellular division of all eggs, as shown in Figure 1. The spindle birefringence in the injected egg decreased, which indicates that the microtubules in the spindle were disassembled.

Shortly after injecting the antibody into metaphase eggs, the spindle shortened and its birefringence decreased (Fig. 2d) and finally disappeared (Fig. 2f), which indicates that the spindle microtubules depolymerized. Although two groups of chromatids separated slightly after injection at metaphase, they formed only

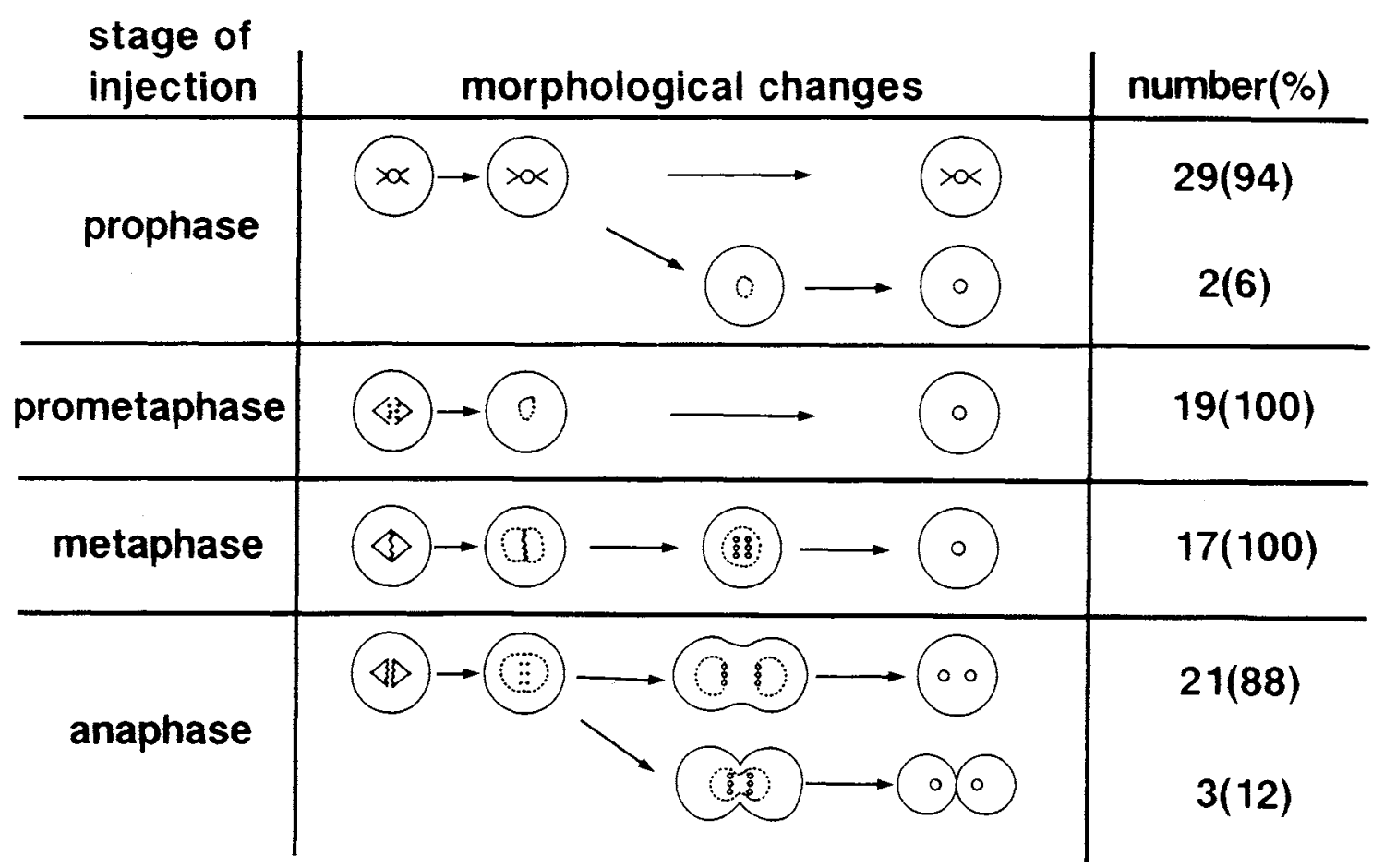

Fig. 1. Results of $\mathrm{YL} 1 / 2$ injection into the dividing $C$. japonicus egg. The pictures schematically indicate morphological changes in eggs, nucleus, chromosomes, or spindle after injection. Morphological changes progress with time from left to right in the figure. 
one nucleus afterward (Fig. $2 \mathrm{~g}, \mathrm{~h}$ ). Furrows did not develop, although some eggs showed such dividing activity as changing from spherical to ellipsoidal shape (Fig. $2 \mathrm{~g}$ ) and recovered their spherical shape (Fig. $2 \mathrm{~h}$ ).

In 21 out of 24 eggs injected at anaphase $(88 \%)$, the cleavage furrow developed, but then regressed (Figs. 1 and $3 \mathrm{c}, \mathrm{d}$ ). The daughter chromosomes formed two nuclei, which came close together and fused into one (Fig. $3 \mathrm{~d})$. In 3 out of 24 injected eggs, the cleavage was completed, which might have resulted from injecting the antibody into these eggs at late anaphase.

Injection of D2D6 antibody. Fourteen out of 26 eggs injected with D2D6 before nuclear envelope disappearance did not develop to prometaphase, and the remaining twelve eggs divided into two (Table I), whereas injection of the control solution caused no significant effect. This inhibition did not seem dependent on the injection time after insemination, the injection volume, or the solution used for injection (ascites fluid or culture supernatant). On the other hand, all 52 eggs in- jected with D2D6 at prometaphase or later completed their division even when a large excess of D2D6 was injected.

To investigate the possibility that D2D6 would bind to microtubules in the egg cytoplasm without microtubule disassembly, FITC-conjugated D2D6 (FITCD2D6) was injected into the sand dollar egg. When the injected egg developed at metaphase, bright fluorescence was not observed in the mitotic apparatus. In some cases, the fluorescence was slightly bright in the yolk-free region of the cell center. Images similar to those of FITC-D2D6-injected eggs were generally observed in the eggs injected with fluorescently labeled proteins such as BSA, $\alpha$-lactoalbumin, and troponin which did not accumulate in the mitotic apparatus (9). These results may be caused by the fact that there is more cytoplasm accessible to soluble proteins in that region than in the other region.

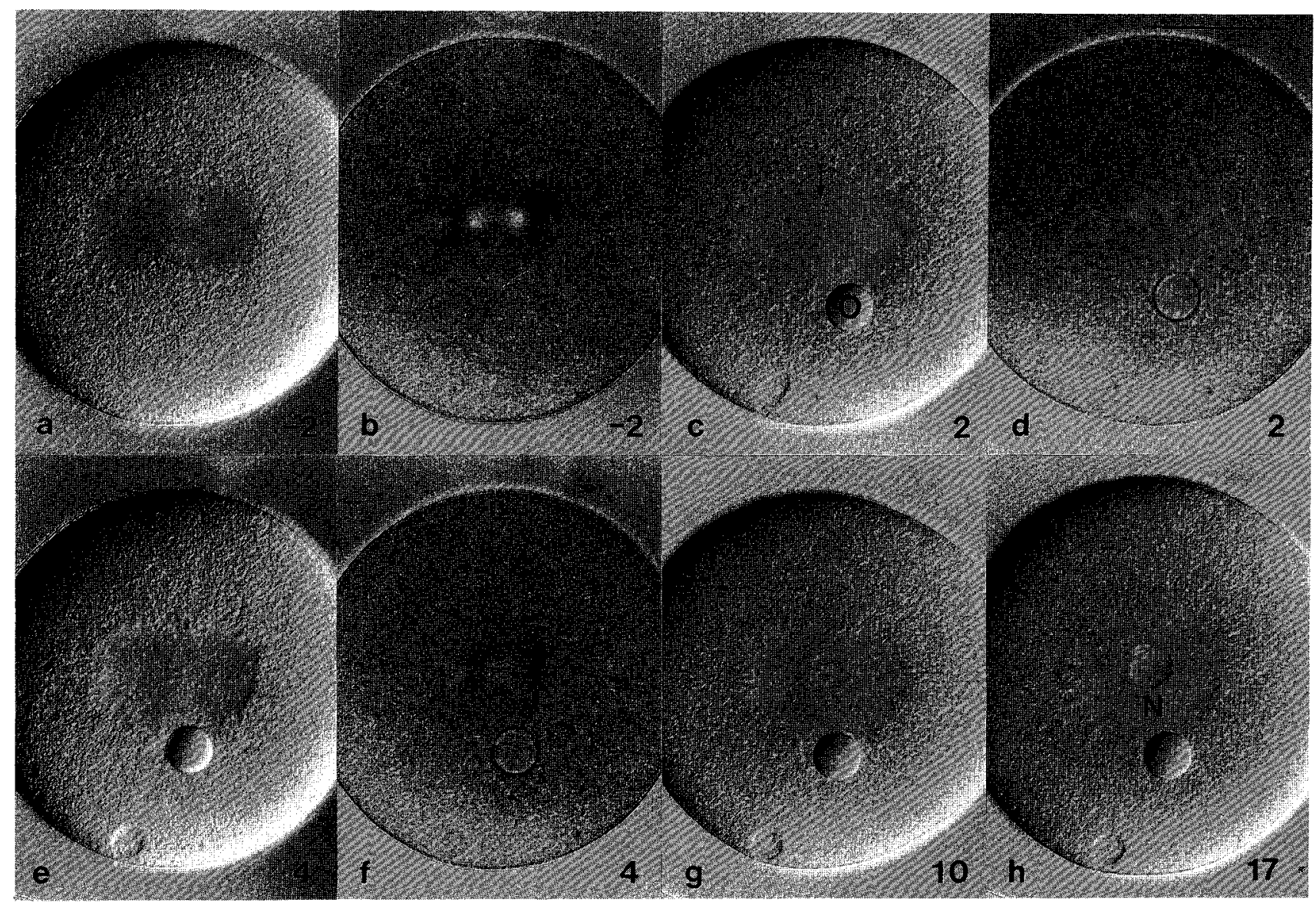

Fig. 2. A sand dollar egg injected with YL1/2 at metaphase. a, c, e, g, and h: Differential interference contrast micrographs. b, d, and f: Polarization micrographs. The numbers indicate the time (minutes) before $(a, b)$ or after injection (c-h). The arrow indicates chromosomes. N: Nucleus; O: Oil drop. The bar shows $50 \mu \mathrm{m}$. 


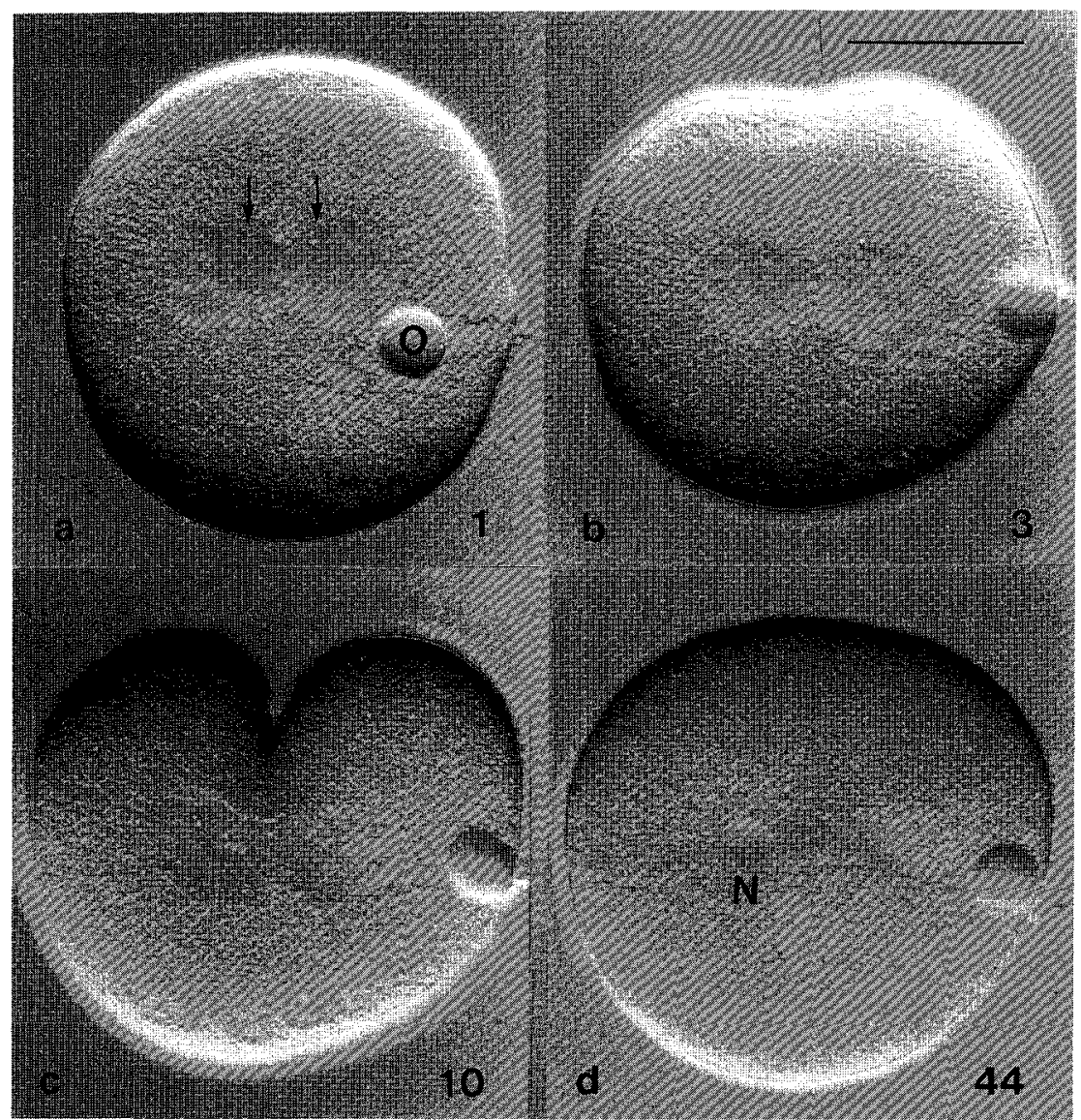

Fig. 3. A sand dollar egg injected with YL1/2 at anaphase. a, b, c, and $\mathrm{d}$ : Differential interference contrast micrographs. The numbers indicate the time (minutes) before (a) or after injection (b-d). Arrows indicate chromosomes. $\mathrm{N}$ is one of two nuclei, and the other is out of focus in $\mathrm{d}$. $\mathrm{O}$ : Oil drop. The bar shows $50 \mu \mathrm{m}$.

\section{DISCUSSION}

In our previous study, YL1/2 strongly reacted with the microtubules of both the spindle and asters in fixed sand dollar eggs (shown by indirect immunofluorescence) and reacted with only $\alpha$-tubulin in the whole egg extract (by Western blot analysis) (13). In the present study, injection of the monoclonal anti-tubulin antibody YL1/2 decreased the spindle birefringence and pre-

Table I. EFfects of D2D6 inJeCtion INTo THE DIVIDing $C$. japonicus EGG.

\begin{tabular}{lcc}
\hline $\begin{array}{l}\text { Stage at the time } \\
\text { of injection }\end{array}$ & $\begin{array}{c}\text { Eggs which failed } \\
\text { to divide }\end{array}$ & $\begin{array}{c}\text { Eggs which divided } \\
\text { into two cells* }\end{array}$ \\
\hline prophase & 14 & 12 \\
prometaphase & 0 & 17 \\
metaphase & 0 & 19 \\
anaphase & 0 & 16 \\
\hline
\end{tabular}

\footnotetext{
* Some of them divided two or more times.
}

vented cleavage, which suggests that YL1/2 reacted with microtubules in the living sand dollar egg as well as in the fixed egg and disassembled the mitotic microtubules. Furthermore, it is thought that inhibition of both nuclear disappearance in eggs injected at prophase and cleavage completion in eggs injected at anaphase may also be owing to microtubule depolymerization.

However, the results in this study were somewhat different from the previously reported findings: microtubules in the CHO cell bundled and, therefore, its division was arrested by injection of YL1/2 (20), and microtubules also bundled in the Drosophila embryo upon injection of YL1/2 (19). These reports show that YL1/2 would not disassemble microtubules in $\mathrm{CHO}$ or Drosophila embryonic cells, but made them bundle as a result of binding to microtubules. The reason is unknown, although there may only be the difference in the cells used for injection, which may indicate the difference in their microtubules. For instance, the amount of tubulin isotypes which reacted with YL1/2 in the sea urchin egg may be greater than that in $\mathrm{CHO}$ and 
Drosophila cells because the content in the sea urchin egg was much more than that in rat brain cells (our preliminary data). Generally, two characteristics of microtubules may cause the difference in the responses to the antibody injection. 1. The composition of tubulin isotypes in microtubules is different and an isotype different from the YL1/2-binding tubulin isotype(s) may govern the responses. 2. The amount of YL1/2-binding tubulin in microtubules is different and the microtubules containing a large amount of $\mathrm{YL} 1 / 2$-binding tubulin may disassemble in response to the injection of YL1/2.

It might be imagined that D2D6 would disassemble spindle microtubules specifically because it stained mainly the spindle. However, that was not the case. Injection of D2D6 did not significantly affect the mitosis of the sand dollar egg. FITC-D2D6 appeared unbound to mitotic microtubules. By immunofluorescence, D2D6 did not stain the mitotic apparatus of the lysed but unfixed egg (our unpublished data). These results suggest that D2D6 would not bind to mitotic microtubules in the living cell, in spite of its binding to them in the fixed cell. However, it still remains possible that (a) D2D6 would bind to them but did not disassemble them in the living cell, as reported by Draber et al. using different antibodies (6), or (b) the accumulation of FITC-D2D6 was not detected because D2D6 reacted with a smaller portion of the total $\alpha$-tubulin content included in the sea urchin eggs compared to $\mathrm{YL} 1 / 2$, as reported previously (13).

In conclusion, some of the antibodies which bind to microtubules in the fixed cell can bind to microtubules in the living cell, but others cannot. Antibody binding to microtubules may cause three different effects on microtubule dynamics in the living cell: (1) it disassembles microtubules (YL1/2, the present study), (2) it bundles microtubules $(19,20)$, or (3) it has no significant effect (6; D2D6, the present study) or some side effects such as the effect on intermediate filaments (3). Therefore, many factors other than the binding specificity of the antibody - such as binding ability to microtubule and tubulin dimer, and binding quantity of tubulin isotypes in the cell-should be considered when investigating the function of microtubules by means of injection of monoclonal anti-tubulin antibody.

Acknowledgments. We thank Dr. G. Matsumoto (Electrotechnical $\mathrm{Lab}$.) for his valuable discussions. We thank also the staff of The Misaki Marine Biological Station for supplying the sand dollars.

This work was supported by Grants-in-Aid from the Ministry of Education, Science, and Culture of Japan to T.A. (01658001) and Y.H. (62540538).

\section{REFERENCES}

1. Arai, T. and Matsumoto, G. (1988). Subcellular localization of functionally differentiated microtubules in squid neurons: Regional distribution of microtubule-associated proteins, and $\beta$-tubulin isotypes. J. Neurochem. 51: 1825-1838.

2. Arai, T., Ichikawa, M., and Matsumoto, G. (1989). Functional differentiation of microtubules localized in the subaxolemmal axoplasm of the squid giant axon. in Proceeding of the 14th Taniguchi International Symposium: Dynamics of Microtubules. (H. Hotani, ed.) The Taniguchi Foundation, pp 260-282.

3. Blose, S.H., Meltzer, D.I., and Feramisco, J.R. (1984). 10$\mathrm{nm}$ filaments are induced to collapse in living cells microinjected with monoclonal and polyclonal antibodies against tubulin. $J$. Cell Biol. 98: 847-858.

4. Cleveland, D.W. (1987). The multitubulin hypothesis revisited. J. Cell Biol. 104: 381-383.

5. De Pennart, H., Houliston, E., and Maro, B. (1988). Posttranslational modifications of tubulin and the dynamics of microtubules in mouse oocytes and zygotes. Biology of the Cell 64: $375-378$.

6. Dráber, P., Dráberová, E., Linhartová, I., and ViKLickÝ, V. (1989). Differences in the exposure of $\mathrm{C}$ - and $\mathrm{N}$-terminal tubulin domains in cytoplasmic microtubules detected with domain-specific monoclonal antibodies. J. Cell Sci. 92: 519-528.

7. Fulton, C. and Simpson, R.A. (1976). Selective synthesis and utilization of flagellar tubulin. The multi-tubulin hypothesis. In Cell Motility. (R. Goldman, T. Pollard, and J.L. Rosenbaum, eds.): New York: Cold Spring Harbor Press, pp. 987-1005.

8. Gundersen, G.G. and Bulinski, J.C. (1986). Distribution of tyrosinated and nontyrosinated $\alpha$-tubulin during mitosis. J. Cell Biol. 102: 1118-1126.

9. Hamaguchi, Y., Toriyama, M., SaKai, H., and Hiramoto, Y. (1985). Distribution of fluorescently labeled tubulin injected into sand dollar eggs from fertilization through cleavage. $J$. Cell Biol. 100: 1262-1272.

10. Hiramoto, Y. (1974). A method of microinjection. Exp. Cell Res. 87: 403-406.

11. Kilmartin, J.V., Wright, B., and Milstein, C. (1982). Rat monoclonal antitubulin antibodies derived by using a new nonsecreting rat cell line. J. Cell Biol. 93: 576-582.

12. LoWry, O.H., Rosebrough, N.J., FARR, A.L., and RANDall, R.J. (1951). Protein measurement with Folin phenol reagent. J. Biol. Chem. 193: 265-555.

13. Oka, M.T., Arai, T., and Hamaguchi, Y. (1990). Heterogeneity of microtubules in dividing sea urchin eggs revealed by immunoffuorescence microscopy: Spindle microtubules are composed of tubulin isotypes different from those of astral microtubules. Cell Motil. and Cytoskeleton 16: 239-250.

14. Piperno, G., LeDizet, M., and Chang, X. (1987). Microtubules containing acetylated $\alpha$-tubulin in mammalian cells in culture. J. Cell Biol, 104: 289-302.

15. Pfeffer, T.A., Asnes, C.F., and Wilson, L. (1976). Properties of tubulin in unfertilized sea urchin eggs. J. Cell Biol. 69: 599-607.

16. Planques, V., Ducommun, B., Bertrand, M.A., Tollon, Y., and WRIGHT, M. (1989). Variation of the immunolabelling of the $\alpha_{1}$-isotubulin in the mitotic spindle of Physarum polycephalum. Protoplasma 148: 120-129.

17. Schatten, G., Simerly, C., Asai, D.J., Szooke, E., Cooke, P., and Schatten, H. (1988). Acetylated $\alpha$-tubulin in microtubules during mouse fertilization and early development. Dev. 
Biol. 130: 74-86.

18. Sterngerger, L.A. (1979). Immunofluorescence in Immunocytochemistry. John Wiley and Sons, New York, pp. 24-58.

19. Warn, R.M., FlegG, L., and Warn, A. (1987). An investigation of microtubule organization and functions in living Drosophila embryos by injection of a fluorescently labeled antibody against tyrosinated $\alpha$-tubulin. J. Cell Biol. 105: 17211730.

20. Wehland, J., Willingham, M.C., and Sandoval, I.V.
(1983). A rat monoclonal antibody reacting specifically with the tyrosylated form of $\alpha$-tubulin. I. Biochemical characterization, effects on microtubule polymerization in vivo and microtubule polymerization and organization in vivo. J. Cell Biol. 97: 1467-1475.

21. WILson, P.J. and Forer, A. (1989). Acetylated $\alpha$-tubulin in spermatogenic cells of the crane fly Nephrotoma suturalis: Kinetochore microtubules are selectively acetylated. Cell Motil. Cytoskeleton 14: 237-250.

(Received for publication, August 9, 1990 